\title{
POLYNOMIALS AND ENTIRE FUNCTIONS: ZEROS AND GEOMETRY OF THE UNIT BALL
}

\author{
Konstantin M. Dyakonov
}

Abstract. We characterize the extreme and exposed points of the unit ball in certain $L^{1}$-spaces of polynomials and entire functions.

\section{Introduction}

Given a Banach space $X$, we write $b(X)$ for its unit ball:

$$
b(X):=\{x \in X:\|x\| \leq 1\} .
$$

As usual, an element $x \in b(X)$ is said to be an extreme point of $b(X)$ if it is not a proper convex combination of two distinct points in $b(X)$. Further, an $x$ in $b(X)$ is called an exposed point of $b(X)$ if there exists a functional $\Phi \in X^{*}$ such that $\|\Phi\|=1$ and the set $\{y \in b(X): \Phi(y)=1\}$ consists of precisely one element, $x$. Of course, every exposed point is extreme, and every extreme point is of norm one.

In this paper, we determine the extreme and exposed points of the unit ball in the following spaces:

- the space $\mathcal{P}_{n}(\mathbf{T})$ of all (analytic) polynomials $p$ of degree $\leq n$, living on the circle $\mathbf{T}:=\{z \in \mathbb{C}:|z|=1\}$ and endowed with the $L^{1}$-norm

$$
\|p\|_{1, \mathbf{T}}:=\frac{1}{2 \pi} \int_{\mathbf{T}}|p(z)||d z|
$$

- the space $\mathcal{P}_{n}(K)$ of all polynomials $p$ of degree $\leq n$, living on a reasonable subset $K$ of the real line $\mathbb{R}$, with norm

$$
\|p\|_{1, K}:=\int_{K}|p(x)| d x
$$

- the space $\mathcal{E}_{a}^{1}$ of all entire functions $f: \mathbb{C} \rightarrow \mathbb{C}$ of exponential type $\leq a$ satisfying

$$
\|f\|_{1, \mathbb{R}}:=\int_{\mathbb{R}}|f(x)| d x<\infty .
$$

Before describing our results, let us take a look at two other classical spaces. The first is $L^{1}$ itself (say, with respect to Lebesgue measure living on some subset of $\left.\mathbb{R}^{d}\right)$. It is well known that $b\left(L^{1}\right)$ has no extreme points, and hence a fortiori no exposed points. Next, consider the Hardy space $H^{1}$ (on $\mathbf{T}$ or on $\mathbb{R}$ ). This

Received January 20, 2000. 
time, the extreme points turn out to be the unit-norm outer functions (see [2] or [6, pp. 157-158]), whereas the exposed points of $b\left(H^{1}\right)$ seem to admit no nice characterization at all (see, however, [11] for some partial results). Now, when passing from $H^{1}$-functions to polynomials or to entire functions in $\mathcal{E}_{a}^{1}$, we actually replace the semiboundedness condition on the spectrum (i.e., on the support of the Fourier transform) by a boundedness condition. This done, the exposed points become far less mysterious, as we are going to show.

The rest of the paper is organized as follows. In Section 2 below, we state a preliminary lemma on extreme and exposed points in subspaces of $L^{1}$. Then we proceed by solving the problem for each of the three spaces in question; this is done in Sections 3, 4, and 5. Basically, our treatment of the three spaces follows the same pattern; however, each case has its own specific features. In particular, $\mathcal{P}_{n}(K)$ differs from $\mathcal{P}_{n}(\mathbf{T})$ in that $K$ may have "endpoints" (in the appropriate sense), while $\mathbf{T}$ has none; the role of these becomes apparent in Section 4. Further, moving on to entire functions, we have to face a new difficulty which stems from the fact that there are nontrivial entire functions of exponential type 0 (while the only polynomials of degree 0 are constants). The resulting complication manifests itself in Theorem 3(B) and is discussed at length in Section 6. That last section also contains some other remarks and a question.

\section{Preliminaries}

The following (known) result will be frequently used in the sequel. When stating it, we write $L_{\mathbb{R}}^{\infty}$ for the set of real-valued functions in $L^{\infty}$.

Lemma 1. Let $(S, \mu)$ be a measure space, and $X$ a closed subspace of $L^{1}(S, \mu)$. Suppose $f \in X$ is a function with $\|f\|_{1}:=\int_{S}|f| d \mu=1$ that vanishes on no set of positive measure. Then

(A) $f$ is an extreme point of $b(X)$ if and only if every function $h \in L_{\mathbb{R}}^{\infty}(S, \mu)$ satisfying $f h \in X$ is a.e. constant.

(B) $f$ is an exposed point of $b(X)$ if and only if every nonnegative measurable function $h$ on $S$ satisfying $f h \in X$ is a.e. constant.

Sketch of the Proof. For part (A), we refer to [5, Chap. V, Sect. 9], where the required fact is established for $X=H^{1}$; the general case is completely similar.

To prove (B), take a $\Phi \in X^{*}$ with $\|\Phi\|=1$. The Hahn-Banach theorem yields

$$
\Phi(g)=\int_{S} \varphi g d \mu, \quad g \in X,
$$

for some $\varphi \in L^{\infty}(S, \mu)$ with $\|\varphi\|_{\infty}=1$. Therefore, the equality $\Phi(g)=1$ occurs for $g \in b(X)$ if and only if $\|g\|_{1}=1$ and $\varphi g=|g|$ almost everywhere. Combining this with the definition of an exposed point leads to the desired conclusion.

We also refer to $[2$, p. 478], where the above reasoning is applied to the case $X=H^{1}$ (without actually using any specific facts about $H^{1}$ ). 
Besides, we need the notation $\mathcal{Z}(f)$ for the zero-set of an entire function $f$; multiple zeros are included in several copies, according to their multiplicities. In particular, if $p$ is a polynomial of degree $m$, then $\mathcal{Z}(p)$ is the $m$-tuple of its zeros.

\section{Polynomials on the circle}

Theorem 1. Let

$$
p(z)=c_{0}+c_{1} z+\ldots+c_{n} z^{n}
$$

be a polynomial with $\|p\|_{1, \mathbf{T}}=1$. Then

(A) $p$ is an extreme point of $b\left(\mathcal{P}_{n}(\mathbf{T})\right)$ if and only if the following two conditions hold:

(i.1) $\left|c_{0}\right|+\left|c_{n}\right| \neq 0$,

(ii.1) $p$ has no pair of symmetric zeros with respect to $\mathbf{T}$.

(B) $p$ is an exposed point of $b\left(\mathcal{P}_{n}(\mathbf{T})\right)$ if and only if $p$ satisfies (i.1), (ii.1), and

(iii.1) the zeros of $p$ lying on $\mathbf{T}$ (if any) are all simple.

We emphasize that the pairs in (ii.1) are nondegenerate pairs consisting of two distinct points (the same applies to conditions (ii.2) and (ii.3) below), so that multiple zeros on $\mathbf{T}$ are allowed in (ii.1). Now (ii.1) and (iii.1) together forbid both degenerate and nondegenerate pairs of symmetric zeros.

Proof. (A) If $c_{0}=0$ and $c_{n}=0$, then both $z p$ and $\bar{z} p(=p / z)$ are in $\mathcal{P}_{n}(\mathbf{T})$, whence $p \cdot \Re z \in \mathcal{P}_{n}(\mathbf{T})$. Similarly, if $p(a)=p(1 / \bar{a})=0$ for some $a \in \mathbf{D} \backslash\{0\}$ (here $\mathbf{D}$ is the open unit disk) then, setting $b(z):=(z-a) /(1-\bar{a} z)$, we have $p b \in \mathcal{P}_{n}(\mathbf{T})$ and $p \bar{b}(=p / b) \in \mathcal{P}_{n}(\mathbf{T})$, whence $p \cdot \Re b \in \mathcal{P}_{n}(\mathbf{T})$. The functions $\Re z$ and $\Re b$ being real and bounded on $\mathbf{T}$, an application of Lemma 1(A) shows that (i.1) and (ii.1) are necessary for $p$ to be an extreme point.

Conversely, let (i.1) and (ii.1) hold. Suppose $h \in L_{\mathbb{R}}^{\infty}(\mathbf{T})$ is a function such that $q:=p h$ is a polynomial of degree $\leq n$. Set

$$
p^{*}(z):=z^{n} \overline{p(1 / \bar{z})}
$$

so that

$$
p^{*}(z)=\bar{c}_{n}+\bar{c}_{n-1} z+\ldots+\bar{c}_{0} z^{n}
$$

and let $q^{*}$ be similarly built from $q$. Since $h$ is real-valued, we have

$$
h=\frac{q}{p}=\frac{\bar{q}}{\bar{p}}=\frac{q^{*}}{p^{*}}
$$

a.e. on T. In particular,

$$
p q^{*}=p^{*} q
$$

a.e. on $\mathbf{T}$, and hence everywhere in $\mathbb{C}$ (indeed, both sides of (3) are polynomials). Now, since conditions (i.1) and (ii.1) together mean that $p$ and $p^{*}$ have no common zeros in $\mathbb{C} \backslash \mathbf{T}$, it follows from (3) that

$$
\mathcal{Z}(p) \backslash \mathbf{T} \subset \mathcal{Z}(q) .
$$


In fact, we also have $\mathcal{Z}(p) \cap \mathbf{T} \subset \mathcal{Z}(q)$, since otherwise the ratio $h=q / p$ would not be essentially bounded on $\mathbf{T}$. Thus,

$$
\mathcal{Z}(p) \subset \mathcal{Z}(q) .
$$

Similarly, we infer from (2) and (3) that

$$
\mathcal{Z}\left(p^{*}\right) \subset \mathcal{Z}\left(q^{*}\right) .
$$

(To keep on the safe side, we recall that multiplicities are taken into account in all of the above inclusions between zero-sets.)

Assume for the moment that $c_{n} \neq 0$. Then the degree of $p$ equals $n$, and since the degree of $q$ is no larger, we deduce from (5) that $\mathcal{Z}(p)=\mathcal{Z}(q)$ and $q / p=$ const. Otherwise, we have $c_{n}=0$ and (i.1) gives $c_{0} \neq 0$, so that $p^{*}$ has degree $n$. The degree of $q^{*}$ being $\leq n$, we then invoke (6) to find that $q^{*} / p^{*}=$ const.

Recalling (2), we eventually conclude, in both cases, that $h=$ const. Now Lemma $1(\mathrm{~A})$ convinces us that $p$ is an extreme point.

(B) Since every exposed point is extreme, (i.1) and (ii.1) are necessary for $p$ to be exposed in $b\left(\mathcal{P}_{n}(\mathbf{T})\right)$. Now if $\zeta_{0} \in \mathbf{T}$ were a multiple zero for $p$ (here and below, multiple means of multiplicity $\geq 2)$, then

$$
h_{0}(z):=-\left(\frac{z+\zeta_{0}}{z-\zeta_{0}}\right)^{2}, \quad z \in \mathbf{T} \backslash\left\{\zeta_{0}\right\},
$$

would be a nonnegative function with the property that $p h_{0} \in \mathcal{P}_{n}(\mathbf{T})$. Therefore, by Lemma 1(B), condition (iii.1) is also necessary.

Conversely, let (i.1), (ii.1) and (iii.1) hold, and assume $h \geq 0$ is a (measurable) function on $\mathbf{T}$ such that $q:=p h \in \mathcal{P}_{n}(\mathbf{T})$. As before, we have then (2), (3) and (4) at our disposal: indeed, these were deduced above from (i.1), (ii.1) and the mere fact that $h$ is real-valued. It follows that the rational function $q / p$ is analytic on $\mathbb{C} \backslash \mathbf{T}$, while by (iii.1) it has at worst several simple poles on $\mathbf{T}$. This in turn implies that $q / p$ belongs to the Hardy space $H^{1-\varepsilon}$ of the disk $\mathbf{D}$, whenever $0<\varepsilon<1$; in particular, $q / p \in H^{1 / 2}$. Since positive $H^{1 / 2}$-functions are constant (see, e.g., [6, Exercise 13(c) in Chap. II]), we conclude that $h=q / p=$ const. In view of Lemma 1(B), this completes the proof.

\section{Polynomials on subsets of the real line}

Given a measurable set $K \subset \mathbb{R}$, we put

$$
K^{*}:=\{x \in \mathbb{R}:|K \cap(x-\varepsilon, x+\varepsilon)|>0 \text { for all } \varepsilon>0\},
$$

where $|\cdot|$ denotes length. Further, we define $K_{0}$ as the set of those $x \in K^{*}$ which have the following property: there is a $\delta=\delta(x)>0$ such that either $|K \cap(x-\delta, x)|=0$ or $|K \cap(x, x+\delta)|=0$. We say that $K$ is essentially bounded if $K^{*}$ is bounded.

Theorem 2. Let $K$ be an essentially bounded subset of $\mathbb{R}$, and suppose $p$ is a polynomial of the form (1) with $\|p\|_{1, K}=1$. Then 
(A) $p$ is an extreme point of $b\left(\mathcal{P}_{n}(K)\right)$ if and only if the following three conditions hold:

(i.2) $c_{n} \neq 0$,

(ii.2) $p$ has no pair of symmetric zeros with respect to $\mathbb{R}$,

(iii.2) the real zeros of $p$ (if any) are all contained in $K^{*}$.

(B) $p$ is an exposed point of $b\left(\mathcal{P}_{n}(K)\right)$ if and only if it satisfies (i.2), (ii.2), and (iii.2) in conjunction with

(iv.2) $p(x) \neq 0$ whenever $x \in K_{0}$, and

(v.2) the real zeros of $p$ (if any) are all simple.

Proof. (A) If (i.2) fails (i.e., if $c_{n}=0$ ), then $x p(x) \in \mathcal{P}_{n}(K)$. Next, if (ii.2) fails (i.e., if $p(w)=p(\bar{w})=0$ for some $w \in \mathbb{C} \backslash \mathbb{R}$ ), then we put $b_{w}(x):=$ $(x-w) /(x-\bar{w})$ and notice that both $p b_{w}$ and $p \bar{b}_{w}$ are in $\mathcal{P}_{n}(K)$; hence also $p \cdot \Re b_{w} \in \mathcal{P}_{n}(K)$. Finally, if (iii.2) fails, then $p(\gamma)=0$ for some $\gamma \in \mathbb{R} \backslash K^{*}$, and so $p(x) \cdot(x-\gamma)^{-1} \in \mathcal{P}_{n}(K)$. The functions $h_{1}(x):=x, h_{2}(x):=\Re b_{w}(x)$ and $h_{3}(x):=(x-\gamma)^{-1}$ being real, nonconstant and essentially bounded on $K$, we see (in light of Lemma 1) that (i.2), (ii.2) and (iii.2) are necessary for $p$ to be extreme in $b\left(\mathcal{P}_{n}(K)\right)$.

Conversely, suppose (i.2), (ii.2) and (iii.2) hold. Assume furthermore that $q:=p h \in \mathcal{P}_{n}(K)$ for some $h \in L_{\mathbb{R}}^{\infty}(K)$. Consider the polynomials

$$
p^{*}(z):=\overline{p(\bar{z})} \quad \text { and } \quad q^{*}(z):=\overline{q(\bar{z})} .
$$

As in the proof of Theorem 1, we arrive at (2) and (3) (with the new $p^{*}$ and $q^{*}$ plugged in). This time (2) holds a.e. on $K$, and (3) everywhere in $\mathbb{C}$. Since (ii.2) tells us that $p$ and $p^{*}$ have no common zeros in $\mathbb{C} \backslash \mathbb{R}$, we deduce from (3) that

$$
\mathcal{Z}(p) \backslash \mathbb{R} \subset \mathcal{Z}(q)
$$

Besides,

$$
\mathcal{Z}(p) \cap \mathbb{R}=\mathcal{Z}(p) \cap K^{*} \subset \mathcal{Z}(q),
$$

where we have first used (iii.2) and then the fact that $h=q / p$ is essentially bounded on $K$. (Indeed, if $x_{0} \in K^{*}$ were a zero of multiplicity $m_{1}$ for $p$ and of multiplicity $m_{2} \in\left[0, m_{1}\right)$ for $q$, then $q / p$ would be essentially unbounded in any neighborhood of $x_{0}$, and hence also on $K$.)

Combining (7) and (8), we get

$$
\mathcal{Z}(p) \subset \mathcal{Z}(q) .
$$

Since the degree of $q$ does not exceed $n$, while (i.2) says the degree of $p$ is precisely $n$, we conclude from $(9)$ that $\mathcal{Z}(p)=\mathcal{Z}(q)$ and $q / p=$ const. Thus, $h=q / p$ must be constant a.e. on $K$, and $p$ is an extreme point.

(B) Of course, (i.2), (ii.2) and (iii.2) are necessary for $p$ to be exposed. Now let $\alpha \in K_{0}$, so that $\alpha$ is an endpoint of some open interval $I$ satisfying $|K \cap I|=0$. Pick a point $\beta \in I$ and put $h(x):=(x-\beta) /(x-\alpha)$. If $p(\alpha)=0$, then

$$
p h \in \mathcal{P}_{n}(K),
$$


while $h \neq$ const and $h \geq 0$ on $\mathbb{R} \backslash I$ (hence a.e. on $K$ ). When coupled with Lemma 1, this shows that (iv.2) is necessary in order that $p$ be exposed in $b\left(\mathcal{P}_{n}(K)\right)$.

Finally, if $\gamma \in \mathbb{R}$ is a multiple zero for $p$, then (10) holds with $h(x)=(x-$ $\gamma)^{-2} \geq 0$, and $p$ fails to be exposed. The necessity of (v.2) is thus established, too.

Conversely, suppose the five conditions (i.2)-(v.2) are fulfilled. Assume also that $q \in \mathcal{P}_{n}(K)$ and

$$
h:=\frac{q}{p} \geq 0 \quad \text { a.e. on } K .
$$

Using (ii.2), we arrive at (7) exactly as before. Further, we claim that the real zeros of $p$ must be contained among those of $q$. Indeed, let $x_{0} \in \mathcal{Z}(p) \cap \mathbb{R}$. By (iii.2) and (iv.2), we have then $x_{0} \in K^{*} \backslash K_{0}$, so that both $K_{\varepsilon}^{-}:=K \cap\left(x_{0}-\varepsilon, x_{0}\right)$ and $K_{\varepsilon}^{+}:=K \cap\left(x_{0}, x_{0}+\varepsilon\right)$ are sets of positive measure whenever $\varepsilon>0$. Now if $x_{0} \notin \mathcal{Z}(q)$, then, by virtue of (v.2), $x_{0}$ would be a simple zero for the function $p / q$. This, however, would contradict (for $\varepsilon$ sufficiently small) the fact that $p / q$ is a.e. nonnegative on both $K_{\varepsilon}^{-}$and $K_{\varepsilon}^{+}$, as ensured by (11).

Consequently, we have (8) and hence also (9) at our disposal. Arguing as in the proof of part (A), we readily conclude that $h=$ const a.e. on $K$, and so $p$ is an exposed point.

Of course, if $K$ is a bounded interval, say $K=(a, b)$, then Theorem 2 applies with $K^{*}=[a, b]$ and $K_{0}=\{a, b\}$. As more sophisticated examples, we mention various Cantor-type sets of positive measure.

Now let us consider essentially unbounded (i.e., not essentially bounded) sets $K$. Among these, we single out the class of essentially semibounded sets. We say that $K$ is essentially semibounded if $K^{*}$ is semibounded (i.e., if either $\inf K^{*}>-\infty$ or $\left.\sup K^{*}<\infty\right)$.

Theorem 2'. Let $K$ be an essentially unbounded subset of $\mathbb{R}$ such that $\int_{K}|x|^{n} d x<\infty$. Suppose $p$ is a polynomial given by (1), with $\|p\|_{1, K}=1$. Then

(A) $p$ is an extreme point of $b\left(\mathcal{P}_{n}(K)\right)$ if and only if it satisfies (ii.2) and (iii.2).

(B) Assume in addition that $K$ is essentially semibounded. Then $p$ is an exposed point of $b\left(\mathcal{P}_{n}(K)\right)$ if and only if it satisfies conditions (i.2)-(v.2).

$\left(\mathrm{B}^{\prime}\right)$ Assume now that $K$ is not essentially semibounded. Then $p$ is an exposed point of $b\left(\mathcal{P}_{n}(K)\right)$ if and only if it satisfies

(i. $\left.2^{\prime}\right)\left|c_{n}\right|+\left|c_{n-1}\right| \neq 0$

in conjunction with (ii.2)-(v.2).

Proof. Let us begin with the "only if" parts. Here, we only have to explain why condition (i.2) is necessary in (B) (observe that it no longer appears in (A)) and why $\left(\mathrm{i} .2^{\prime}\right)$ is necessary in $\left(\mathrm{B}^{\prime}\right)$. The necessity of the other conditions involved is verified as in Theorem 2 . 
If $K$ is essentially semibounded, then, for a suitable $a \in \mathbb{R}$, the function $h_{a}(x):=x-a$ is either a.e. positive or a.e. negative on $K$. Now if (i.2) fails, then (10) holds with $h= \pm h_{a}$, and $p$ fails to be exposed.

Similarly, for generic $K$ 's (in particular, for the ones figuring in $\left(\mathrm{B}^{\prime}\right)$ ), the failure of (i. $\left.2^{\prime}\right)$ would imply (10) with $h(x)=x^{2}$.

The "if" part of (B) is established exactly as in the preceding theorem, while the proofs of the "if" parts of $(\mathrm{A})$ and $\left(\mathrm{B}^{\prime}\right)$ mimic those of $(\mathrm{A})$ and $(\mathrm{B})$ in Theorem 2. Namely, arguing as before, we reach (9) and infer that $q / p$ is a polynomial. This done, the rest of (A) follows from the fact that a polynomial which is bounded on an unbounded set must be constant. Similarly, to complete the proof of $\left(\mathrm{B}^{\prime}\right)$ we invoke the following fact: If a polynomial is positive on a real set for which both $+\infty$ and $-\infty$ are cluster points, then it is either constant or of degree $\geq 2$.

Among the admissible $K$ 's to which Theorem $2^{\prime}$ applies, let us mention countable unions of intervals with suitably decreasing lengths. For such a $K$, it may well happen that $K^{*}=\mathbb{R}$ and $K_{0}=\emptyset$, in which case conditions (iii.2) and (iv.2) disappear from (A) and $\left(\mathrm{B}^{\prime}\right)$. Consider, as an example, the set $K=\bigcup_{j=1}^{\infty}\left(x_{j}-r_{j}, x_{j}+r_{j}\right)$, where $\left\{x_{j}\right\}$ is an enumeration of the rational numbers and $r_{j}=2^{-j}\left(1+\left|x_{j}\right|\right)^{-n}$.

\section{Entire functions of exponential type}

Before stating the result, we introduce the notation $\sigma(f)$ for the type of an entire function $f$ (with respect to order 1 ). Thus

$$
\sigma(f):=\varlimsup_{z \rightarrow \infty} \frac{\log |f(z)|}{|z|},
$$

and the space $\mathcal{E}_{a}^{1}$ consists of (the traces on $\mathbb{R}$ of) those entire functions $f$ for which $\sigma(f) \leq a$ and $\left.f\right|_{\mathbb{R}} \in L^{1}(\mathbb{R})$.

Theorem 3. Let $a>0$, and let $f \in \mathcal{E}_{a}^{1}$ satisfy $\|f\|_{1, \mathbb{R}}=1$. Then

(A) $f$ is an extreme point of $b\left(\mathcal{E}_{a}^{1}\right)$ if and only if the following conditions hold:

(i.3) $\sigma(f)=a$,

(ii.3) $f$ has no pair of symmetric zeros with respect to $\mathbb{R}$.

(B) $f$ is an exposed point of $b\left(\mathcal{E}_{a}^{1}\right)$ if and only if it satisfies (i.3) and (ii.3) in conjunction with

(iii.3) the real zeros of $f$ (if any) are all simple, and

(iv.3) $\int_{-\infty}^{\infty}|f(x)| h(x) d x=\infty$ whenever $h$ is a nonconstant entire function such that $\sigma(h)=0$ and $h \geq 0$ on $\mathbb{R}$.

Much of the proof will be similar to what was done in preceding sections, but this time we also need some facts about entire functions of the Cartwright class. This class, denoted in what follows by Cart, consists of all entire functions $f$ 
satisfying $\sigma(f)<\infty$ and

$$
\int_{\mathbb{R}} \frac{\log ^{+}|f(t)|}{1+t^{2}} d t<\infty
$$

It is easy to see that $\bigcup_{a>0} \mathcal{E}_{a}^{1} \subset$ Cart. Further, with an entire function $f$ we associate the quantities $\sigma_{+}(f)$ and $\sigma_{-}(f)$, defined by

$$
\sigma_{ \pm}(f)=\varlimsup_{y \rightarrow \pm \infty} \frac{\log |f(i y)|}{|y|} .
$$

Lemma 2. (a) If $f_{1}, f_{2} \in$ Cart, and if the ratio $f_{1} / f_{2}$ is an entire function, then it is in Cart.

(b) If $f_{1}$ is entire and of exponential type, and if $f_{2} \in$ Cart, then $\sigma_{+}\left(f_{1} f_{2}\right)=$ $\sigma_{+}\left(f_{1}\right)+\sigma_{+}\left(f_{2}\right)$ and $\sigma_{-}\left(f_{1} f_{2}\right)=\sigma_{-}\left(f_{1}\right)+\sigma_{-}\left(f_{2}\right)$.

(c) If $f \in$ Cart, then $\sigma(f)=\max \left\{\sigma_{+}(f), \sigma_{-}(f)\right\}$.

In fact, part (a) follows at once from Krein's characterization of the Cartwright class; see [7, p. 192], or [9, p. 115]. For part (b), see Theorem 4 on p. 118 of [9] (where more is proved). Finally, a proof of (c) can be found on p. 194 of [7].

Proof of Theorem 3. (A) If $\sigma(f)<a$, then we can find an $\varepsilon>0$ such that $f(x) \cdot \cos \varepsilon x \in \mathcal{E}_{a}^{1}$. Further, if $f(w)=f(\bar{w})=0$ for some $w \in \mathbb{C} \backslash \mathbb{R}$, then we put $b_{w}(x):=(x-w) /(x-\bar{w})$ and note that both $f b_{w}$ and $f \bar{b}_{w}\left(=f / b_{w}\right)$ are in $\mathcal{E}_{a}^{1}$ (the functions involved are regarded as living on $\mathbb{R}$ ); hence also $f \cdot \Re b_{w} \in \mathcal{E}_{a}^{1}$. Since the arising multipliers $\cos \varepsilon x$ and $\Re b_{w}$ are real, nonconstant and bounded on $\mathbb{R}$, we see, with the help of Lemma 1, that (i.3) and (ii.3) are necessary for $f$ to be an extreme point.

Conversely, let (i.3) and (ii.3) hold, and suppose $h \in L_{\mathbb{R}}^{\infty}(\mathbb{R})$ is such that

$$
g:=f h \in \mathcal{E}_{a}^{1} .
$$

Following the strategy employed in the proofs of Theorems 1 and 2, we put

$$
f^{*}(z):=\overline{f(\bar{z})} \quad \text { and } \quad g^{*}(z):=\overline{g(\bar{z})},
$$

so that $f^{*}, g^{*} \in \mathcal{E}_{a}^{1}$. We have then

$$
h=\frac{g}{f}=\frac{\bar{g}}{\bar{f}}=\frac{g^{*}}{f^{*}} \quad \text { a.e. on } \mathbb{R} .
$$

Hence $f g^{*}=f^{*} g$ on $\mathbb{R}$, as well as everywhere in $\mathbb{C}$. This last equality yields

$$
\mathcal{Z}(f) \backslash \mathbb{R} \subset \mathcal{Z}(g)
$$

because (ii.3) ensures that $f$ and $f^{*}$ have no common zeros in $\mathbb{C} \backslash \mathbb{R}$. Besides,

$$
\mathcal{Z}(f) \cap \mathbb{R} \subset \mathcal{Z}(g),
$$

since otherwise the ratio $g / f$ would not be essentially bounded on $\mathbb{R}$. Combining (13) and (14), we get

$$
\mathcal{Z}(f) \subset \mathcal{Z}(g),
$$


whence it follows that $g / f=h$ is an entire function. (Strictly speaking, we use the latter formula to extend $h$ from $\mathbb{R}$ to the whole of $\mathbb{C}$, and we now know that the extension is holomorphic on $\mathbb{C}$.) Moreover, Lemma 2(a) yields $h \in$ Cart, because both $f$ and $g$ are in Cart.

Finally, we claim that $\sigma(h)=0$. To see why, recall first that $h$ is real on $\mathbb{R}$, and so

$$
h(z)=\overline{h(\bar{z})}, \quad z \in \mathbb{C} .
$$

Using (12) in conjunction with Lemma 2(b), we now find that

$$
\sigma_{+}(f)+\sigma_{+}(h)=\sigma_{+}(g) \leq \sigma(g) \leq a,
$$

whence

$$
\sigma_{+}(h) \leq a-\sigma_{+}(f)
$$

Similarly, we obtain

$$
\sigma_{-}(h) \leq a-\sigma_{-}(f)
$$

Since

$$
\sigma_{+}(h)=\sigma_{-}(h)=\sigma(h) \geq 0
$$

(thanks to (16) and Lemma 2(c)), while either $\sigma_{+}(f)$ or $\sigma_{-}(f)$ equals $a$ (thanks to (i.3) and Lemma 2(c) again), it follows from (17) and/or (18) that $\sigma(h)=0$.

It is a well-known consequence of the Phragmén-Lindelöf theorem that an entire function of zero exponential type must be constant if it is bounded on a line. Thus $h=$ const, and $f$ is an extreme point.

(B) To prove the "only if" part, it suffices to show that (iii.3) and (iv.3) are necessary in order that $f$ be exposed. To see why (iii.3) is needed, assume $x_{0} \in \mathbb{R}$ is a multiple zero for $f$ and put $h_{0}(x):=\left(x-x_{0}\right)^{-2}$; we have then

$$
f h \in \mathcal{E}_{a}^{1}
$$

with $h=h_{0} \geq 0$, and $f$ fails to be exposed. Finally, (iv.3) is needed because (19) holds whenever $h$ is entire, of zero exponential type, and such that $f h \in L^{1}(\mathbb{R})$. (In both cases, we have Lemma 1 in mind.)

We now turn to the "if" part. Given that (i.3)-(iv.3) are fulfilled, suppose (12) holds with some nonnegative (possibly unbounded) function $h$ on $\mathbb{R}$. Proceeding as in part (A) above, we reach (13) without trouble. The next step is to verify (14), but the argument is now different. Namely, $x_{0} \in \mathcal{Z}(f) \cap \mathbb{R}$ implies $x_{0} \in \mathcal{Z}(g)$, because otherwise the ratio $g / f=h$ would have a simple pole at $x_{0}$ (due to (iii.3)), contradicting the fact that $h \geq 0$ in a neighborhood of $x_{0}$.

Now that (14) is established, we readily arrive at (15) and conclude that $h$ extends to the whole plane as an entire function. Furthermore, arguing as before, we find that $h$ is of exponential type 0 . That $h=$ const now follows by (iv.3). An application of Lemma 1 completes the proof. 


\section{Concluding remarks and a question}

(1) At first sight, condition (iv.3) in Theorem 3 seems to be quite implicit. Notice, however, that (iv.3) is only concerned with the behavior of $f$ at $\infty$. The condition roughly means that $\left.f\right|_{\mathbb{R}}$ "has no multiple zero at $\infty$ " (i.e., $f$ does not decrease too rapidly along the real line); thus (iv.3) can be viewed as an extension of (iii.3). To make our point clearer, let us consider the following two conditions:

(iv. $3^{\prime}$ ) There is an $A>0$ such that inf $\left\{x^{2}|f(x)|: x \in \mathbb{R},|x| \geq A\right\}>0$;

(iv. $\left.3^{\prime \prime}\right) \int_{-\infty}^{\infty} x^{2}|f(x)| d x=\infty$.

We claim that the implications

$$
\left(\mathrm{iv} .3^{\prime}\right) \Longrightarrow(\mathrm{iv} .3) \Longrightarrow\left(\mathrm{iv} .3^{\prime \prime}\right)
$$

hold true. Indeed, it is obvious that (iv.3) implies (iv. $3^{\prime \prime}$ ), since $x^{2}$ is eligible as one of the functions $h(x)$ figuring in (iv.3). To see that (iv. $\left.3^{\prime}\right)$ implies (iv.3), suppose $f h \in L^{1}(\mathbb{R})$ for some entire function $h$ such that $\sigma(h)=0$ and $h \geq 0$ on $\mathbb{R}$. Since $x^{2}|f(x)| \geq c>0$ for $|x| \geq A$, we have

$$
\int_{\mathbb{R} \backslash(-A, A)} \frac{h(x)}{x^{2}} d x \leq \frac{1}{c} \int_{\mathbb{R}}|f(x)| h(x) d x<\infty .
$$

Now if $h$ is nonconstant, then $\mathcal{Z}(h)$ contains at least two (possibly equal) elements, say $\lambda_{1}$ and $\lambda_{2}$. The function $h(z) /\left[\left(z-\lambda_{1}\right)\left(z-\lambda_{2}\right)\right]$ is therefore entire, of exponential type 0 , and belongs to $L^{1}(\mathbb{R})$ (as implied by (21)). This, however, is only possible if $h \equiv 0$.

Condition (iv. $3^{\prime}$ ) makes it easy to produce examples of exposed points in $b\left(\mathcal{E}_{a}^{1}\right)$. One such example is

$$
f(z)=c_{0} \frac{i+\sin a z}{\left(z-z_{1}\right)\left(z-z_{2}\right)}
$$

where $c_{0}$ is a normalizing constant factor and $z_{1,2}$ are some fixed zeros of the numerator.

On the other hand, none of the two implications in (20) can be reversed.

(2) Yet another class of examples arises as follows. Let $\left\{\lambda_{n}\right\}$ be a sequence of pairwise distinct real numbers such that the system of exponentials $\left\{\exp \left(i \lambda_{n} x\right)\right\}$ is both complete and minimal in $L^{2}(-a, a)$. Consider the canonical product

$$
\varphi(z):=\lim _{R \rightarrow \infty} \prod_{\left|\lambda_{n}\right| \leq R}\left(1-\frac{z}{\lambda_{n}}\right)
$$

(if one of the $\lambda$ 's is zero, replace the corresponding factor by $z$ ) and set

$$
f(z)=f_{j, k}(z):=\frac{\varphi(z)}{\left(z-\lambda_{j}\right)\left(z-\lambda_{k}\right)}
$$

where $j$ and $k$ are any two distinct indices. It is known (see $[9$, Theorem 4 , p. 134],) that $\varphi$ is of exponential type $a$ and that $\varphi(x) /(1+|x|) \in L^{2}(\mathbb{R})$, while $\varphi h \notin L^{2}(\mathbb{R})$ for each nonzero entire function $h$ with $\sigma(h)=0$. From this, it 
follows easily that $f$ is in $\mathcal{E}_{a}^{1}$ and satisfies the four conditions of Theorem 3 . When suitably normalized, it therefore becomes an exposed point of $b\left(\mathcal{E}_{a}^{1}\right)$.

A similar construction is possible with complex $\lambda_{n}$ 's. In connection with completeness and minimality of exponential systems, see e.g. [8] and [10].

(3) Condition (iv.3) remains intact if the functions $h$ appearing there are assumed to be in the Cartwright class. On the other hand, given $h \in$ Cart and $a>0$, the Beurling-Malliavin multiplier theorem (see [1], [7], [8], or [10]) enables us to find a nonzero $f \in \mathcal{E}_{a}^{1}$ such that $f h \in L^{1}(\mathbb{R})$. Thus, the $f$ 's satisfying condition (iv.3) are precisely the ones which are not multipliers, in this sense, for any nontrivial positive $h \in$ Cart with $\sigma(h)=0$.

(4) One can extend Theorems 1 and 3 to the case where the underlying support set ( $\mathbf{T}$ or $\mathbb{R}$, respectively) is replaced by a certain subset $K$ thereof. That would entail additional conditions on the zeros, similar to those appearing in Theorems 2 and $2^{\prime}$. When dealing with entire functions, it seems reasonable to restrict attention to the so-called relatively dense sets $K \subset \mathbb{R}$, since the arising $L^{1}(K)$-norms on $\mathcal{E}_{a}^{1}$ are then equivalent to the original one. (We refer to $[7$, pp. 113-115], for the notion of a relatively dense set and for the fact just stated.) Our results are also adjustable for the case of weighted $L^{1}$-norms on $\mathbf{T}$ and $\mathbb{R}$; however, it is only the support of the weight that actually matters.

(5) In [3], we described the extreme points of the unit ball in a generic subspace $X \subset H^{1}$ that coincides with the kernel of some Toeplitz operator. (A characterization of such $X$ 's can be found in [4].) The (A) parts of our current Theorems 1 and 3 - but not the other results above - fit into that framework and could have been deduced from Theorem 6 of [3]. In the present paper, we were primarily concerned with exposed points. However, it was our choice to explicitly derive the relevant facts about extreme points, too, so as to make the presentation self-contained.

(6) A question, to conclude with: What happens in higher dimensions (say, on $\mathbf{T}^{d}$ and $\left.\mathbb{R}^{d}\right)$ ?

\section{Acknowledgments}

The author was partially supported by Grant 99-01-00103 from the Russian

Foundation for Fundamental Research and by a fellowship from La Comisión Interministerial de Ciencia y Tecnología (Spain).

\section{References}

[1] A. Beurling and P. Malliavin, On Fourier transforms of measures with compact support, Acta Math. 107 (1962), 291-309.

[2] K. de Leeuw and W. Rudin, Extreme points and extremum problems in $H_{1}$, Pacific J. Math. 8 (1958), 467-485.

[3] K.M. Dyakonov, Interpolating functions of minimal norm, star-invariant subspaces, and kernels of Toeplitz operators, Proc. Amer. Math. Soc. 116 (1992), 1007-1013. 
[4] Kernels of Toeplitz operators via Bourgain's factorization theorem, J. Funct. Anal. 170 (2000), 93-106.

[5] T.W. Gamelin, Uniform Algebras, Prentice Hall, Englewood Cliffs, NJ, 1969.

[6] J.B. Garnett, Bounded Analytic Functions, Academic Press, New York, 1981.

[7] V. Havin and B. Jöricke, The Uncertainty Principle in Harmonic Analysis, SpringerVerlag, Berlin, 1994.

[8] P. Koosis, The Logarithmic Integral, Vol. II, Cambridge Univ. Press, Cambridge, 1992.

[9] B.Ya. Levin, Lectures on Entire Functions, AMS Transl. of Math. Monographs, Vol. 150, Amer. Math. Soc., Providence, RI, 1996.

[10] R.M. Redheffer, Completeness of sets of complex exponentials, Adv. in Math. 24 (1977), $1-62$.

[11] D. Sarason, Exposed points in $H^{1}$, I, Oper. Theory: Adv. Appl., 41, Birkhäuser, Basel, 1989, pp. 485-496; Exposed points in $H^{1}$, II, Oper. Theory: Adv. Appl., 48, Birkhäuser, Basel, 1990, pp. 333-347.

Departament de Matemàtica Aplicada i Anàlisi, Universitat de Barcelona, Gran Via 585, E-08071 BARCELONA, SPAin

E-mail address: dyakonov@mat.ub.es

Steklov Institute of Mathematics, St. Petersburg Branch (POMi), Fontanka 27, St. Petersburg, 191011, Russia 